\title{
Differential Potencies for Endogenous Dynorphins Indicate Functional Selectivity at the Delta Opioid Receptor
}

\author{
Keith M. Olson ${ }^{1}$, Justin Lavigne ${ }^{2}$, John Streicher ${ }^{2}$, Frank Porreca ${ }^{3 *}$, and \\ Victor J. Hruby ${ }^{1}$ \\ ${ }^{1}$ University of Arizona, Dept. of Chemistry, Tucson, 85721, AZ, USA, ${ }^{2}$ University of New England, \\ Dept. of Biomedical Sciences, Biddeford, ME, 04005, USA; ${ }^{3}$ University of Arizona, \\ Dept. of Pharmacology, Tucson, AZ, 85721, USA
}

\section{Introduction}

Opioid receptors (ORs) are G-protein Coupled Receptors (GPCRs), which mediate analgesia, tolerance, withdrawal, GI transit. Classically, ORs couple inhibitory $\mathrm{G}_{\mathrm{i} / \mathrm{o}}$ proteins and recruit $\beta$ arrestin - a multifaceted scaffold molecule implicated in opioid mediated effects including tolerance, constipation, dysphoria and naseua [1,2]. Upon activation $\beta$-arrestin and $G \alpha_{\mathrm{i} / \mathrm{o}}$ induce downstream signaling responses such as reduced cAMP levels. Recent drug discovery efforts identified several functionally selective exogenous opiates which prefer certain signaling pathways at a given receptor such as $G \alpha$ stimulation - to others - such as $\beta$-arrestin recruitment and generate desired pharmacological properties $[3,4]$. Noting that most of the $20+$ endogenous opioid peptides are nonselective and some opiates display functional selectivity, two important points emerge. First, endogenous and exogenous ligands, such as those used during studies, do not necessarily generate the same effects. Second, two different endogenous opioid peptides may differentially activate a given receptor. Dynorphin A (DynA) and Dynorphin B (DynB) are considered $\kappa$ OR agonists, despite binding to the $\delta \mathrm{OR}$ at $1.29 \mathrm{nM}$ and $3.39 \mathrm{nM}$ [1], respectively. The Dynorphins start with the 5 amino acid Leuenkephalin (Leu-Enk) sequence - YGGFL - traditionally considered a $\delta O R$ agonist followed by distinct $C$-terminal sequences. Thus, we ask: Do Dynorphin A (1-17), Dynorphin B (1-13) and Leuenkephalin induce functionally selective signaling at the $\delta \mathrm{OR}$ ?

\section{Results and Discussion}

We assessed DynA, DynB and Leu-Enk signaling for two ubiquitous GPCR signals - G $\alpha$ activation and $\beta$-arrestin recruitment - in vitro. GTP $\gamma \mathrm{S}$ stimulation assays were performed using CHO cells expressing the $\delta \mathrm{OR}$, analogous to previous reports [5]. $\beta$-Arrestin- 2 recruitment assays (DiscoveRx) were performed by manufacturer's protocol. We found DynA, DynB and Leu-Enk display different potency rank orders for $\mathrm{G} \alpha$

Table 1. In Vitro Signaling Profile of Endogenous Opioids at $\delta O R$. DynA and DynB show distinct rank orders for arrestin-recruitment, GTPgS and cAMP; in addition DynA and DynB show distinct efficacies for cAMP inhibition relative to Leu-Enk. $\beta$-arrestin2 recruitment $(n=1), c A M P(n=3)$ and GTPyS $(n=2)$ assays performed as referenced in text.

\begin{tabular}{lcccccc}
\hline \multirow{2}{*}{ Ligand } & \multicolumn{3}{c}{$\beta$-arrestin } & \multicolumn{2}{c}{ Recruitment } & \multicolumn{2}{c}{ GTPS } & \multicolumn{2}{c}{ cAMP } \\
& EC 50 & Emax & EC 50 & Emax & EC 50 & Emax \\
\hline Dynorphin A(1-17) & 83 & 1.0 & 488 & 1.04 & 21 & 0.54 \\
Dynorphin B(1-13) & 330 & 1.0 & 383 & 1.04 & 122 & 0.52 \\
Leu-Enkephalin & 17 & 1.0 & 1.86 & 1.00 & 5.3 & 1.00 \\
\hline
\end{tabular}
activity and $\beta$-arrestin recruitment at the $\delta \mathrm{OR}$ (Table 1). DynB more potently activates $\mathrm{G} \alpha$ signaling than $\beta$-arrestin2 recruitment with EC50 values of $83 \mathrm{nM}$ and 230 $\mathrm{nM}$, respectively. In contrast, DynA more potently recruits $\beta$-arrestin than DynB with EC50 values of 83 and $328 \mathrm{nM}$, respectively (Table 1). A reversal in rank order between DynA and DynB likely indicates functional selectivity, such that each 


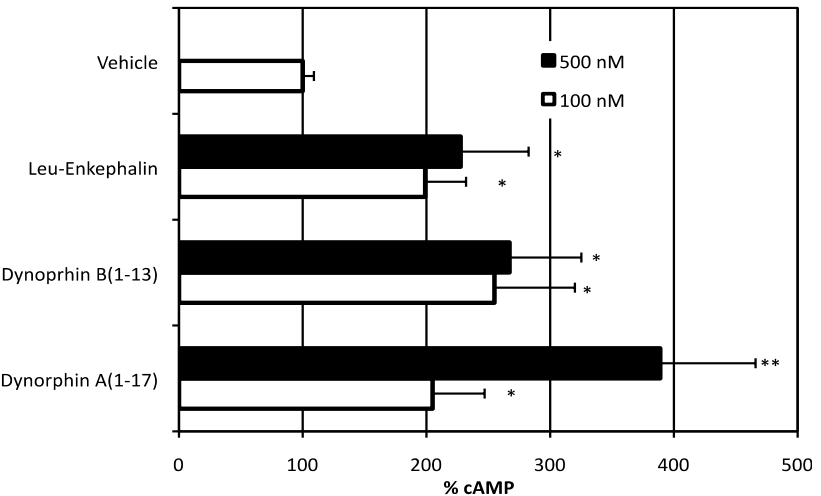

Figure 1. SOR Mediated AC Super Activation. Cells were treated with vehicle or ligand for 24 hours - refreshing media every 8 hours - then AC was stimulated with $10 \mathrm{mM}$ forskolin. Percentage of basal cAMP increase relative to vehicle was graphed and analyzed with a two-taliled t-test $* p<.05, * * p<.01$. ligand likely induces distinct $\delta \mathrm{OR}$ conformations. Thus a modest 3 -fold potency difference, may lead to more pronounced differences downstream.

Next we probed each ligand for forskolin-stimulated cAMP inhibition - a well-established G $\alpha$ dependent pathway [6]. Acute treatment of DynA and Leu-Enk yield $\mathrm{IC}_{50}$ values of $21 \mathrm{nM}$ and $5 \mathrm{nM}$, respectively (Table 1). However, DynB exhibits a $\sim 10$ fold less potent response $\left(\mathrm{IC}_{50}=122 \mathrm{nM}\right)$. DynB is a less potent agonist in the forskolin induced cAMP inhibition compared to DynA and Leu-Enk, whereas all display similar potency in the GTP $\gamma \mathrm{S}$ assay. Based on differential cAMP inhibition potencies and efficacies, we predicted these peptides would differentially effect receptor mediated $\mathrm{G} \alpha$ regulation.

At the $\delta \mathrm{OR}$, chronic opioid treatment causes a compensatory increase in basal cAMP levels, referred to as Adenylyl Cyclase (AC) super activation. AC super activation may play a physiological role in developing opioid tolerance, dependence and withdrawal [5,6]. Chronic treatment with DynA led to greater AC super activation than treatment with DynB or Leu-Enk, which similarly induce AC super activation (Figure 1). This upregulation in forskolin-stimulated cAMP indicates that DynA and DynB induce distinct receptor regulatory events. Differences in AC super activation by endogenous ligands could be important in the physiological roles of disease and require further in vivo study. Thus, in vitro assays show endogenous opioid peptides with differing (or absent) $C$-terminal tails induce distinct changes in the activity of these peptides. Therefore, we show three endogenous opioids induce distinct signaling and regulatory outcomes in vitro. Further studies are required to understand the differences in $\mathrm{G} \alpha$ activation, other cellular regulatory changes and potential uses as a peptide scaffold for drug design.

\section{Acknowledgments}

This work was supported by the U.S. Public Health Services, NIH, and NIDA (P01DA006284).

\section{References}

1. Lamb, K., Tidgewell, K., Simpson, D.S., Bohn, L.M., Prisinzona, T.E. Drug and Alcohol Dependence 121, 181-188 (2012), http://dx.doi.org/10.1016/j.drugalcdep.2011.10.026

2. Raehal, K.M., Walker, J.K.L., Bohn, L.M. J. Pharma. Exper. Therap. 314, 1195-1201 (2005), http://dx.doi.org/10.1124/jpet.105.087254

3. Lamb, K., Tidgewell, K., Simpson, D.S., Bohn, L.M., and Prisinzona, T.E. Drug and Alcohol Dependence. 121, 181-188 (2012), http://dx.doi.org/10.1016/j.drugalcdep.2011.10.026

4. Schmid, C.L., Streicher, J.M., Groer, C.E., Munro, T.A., Zhou, L., Bohn, L.M. (2013). J. Biol. Chem. 288(31):22387-98, http://dx.doi.org/10.1074/jbc.M113.476234

5. Varga, E.V., Rubenzik, M., Grife, V., Sugiyama, M., Stropova, D., Roeske, W.R., Yamamura, H.I. Eur. J. Pharma. 451, 101-102 (2002), http://dx.doi.org/10.1016/S0014-2999(02)02220-3

6. Varga, E.V., Rubenzik, M.K., Stropova, D., Sugiyama, M., Grife, V.., Hruby, V.J., Yamamura, H.I. J. Pharma. Exper. Therap. 306, 109-115 (2003), http://dx.doi.org/10.1124/ipet.103.049643 